\title{
ASPECTOS TEMPORAIS AUDITIVOS EM ADOLESCENTES DO 6 ANO DO ENSINO FUNDAMENTAL
}

\author{
Temporal aspects of hearing in adolescents from the $6^{\text {th }}$ year \\ of elementary education
}

\author{
Sulamita da Silva Marcelino Terto ${ }^{(1)}$, Stela Maris Aguiar Lemos ${ }^{(2)}$
}

\begin{abstract}
RESUMO
Objetivo: esclarecer a relação entre desempenho escolar e aspectos temporais auditivos. Método: estudo descritivo-transversal com amostra de conveniência, composta por 82 estudantes, na faixa etária de 11 a 13 anos, sendo 43 do sexo feminino e 38 do sexo masculino do $6^{\circ}$ ano de uma escola de financiamento privado na Região Metropolitana de Belo Horizonte. Cada estudante respondeu a um formulário de caracterização da amostra, realizou o Teste de Desempenho Escolar e foi submetido aos testes que avaliam os aspectos temporais auditivos: Teste Padrão de Duração, Teste Padrão de Frequência e Teste Gap-in-Noise. Resultados: os testes que avaliam os aspectos temporais auditivos não sofreram influência relacionada às variáveis sexo, idade, realização de atividades de musicalização e preferência manual. Com exceção da variável "acompanhamento fonoaudiológico" que apresentou significância estatística no teste Gap-in-Noise. Os subtestes do Teste de Desempenho Escolar que exerceram maior influencia no desempenho nos testes Padrão de Duração e Padrão de Frequência foram Escrita, seguida de Leitura e Aritmética. Conclusão: a análise dos dados revelou que há correlação entre desempenho escolar e aspectos temporais auditivos. Cabe ressaltar que os testes que avaliam a ordenação temporal complexa (Teste Padrão de Frequência e Teste Padrão de Duração) são influenciados pelo desempenho no Teste de Desempenho Escolar. Porém o mesmo não ocorre com o teste que avalia resolução temporal (Gap-in-Noise).
\end{abstract}

DESCRITORES: Audição; Percepção Auditiva; Testes Auditivos; Estudantes

\section{INTRODUÇÃO}

A integridade auditiva é um pré-requisito para um efetivo processo de ensino-aprendizagem. Isso porque, a audição desempenha importante papel na aquisição e desenvolvimento da linguagem, além da contribuição decisiva e preponderante na comunicação. Sabe-se que para um satisfatório desempenho, é necessário a integridade do sistema auditivo nos níveis periférico e central. Evidências sugerem que um elemento subjacente a inúmeras

(1) Acadêmica do curso de Fonoaudiologia da Universidade Federal de Minas Gerais - UFMG, Belo Horizonte, MG, Brasil.

(2) Fonoaudióloga; Professora Adjunto do Curso de Fonoaudiologia da Universidade Federal de Minas Gerais, UFMG, Belo Horizonte, MG, Brasil; Doutora em Distúrbios da Comunicação Humana (Fonoaudiologia) pela Universidade Federal de São Paulo, UNIFESP, Brasil.

Conflito de interesses: inexistente capacidades do processamento auditivo, são as habilidades do processamento temporal, uma vez que muitas das características que englobam a informação auditiva são de alguma forma, influenciadas pelo tempo ${ }^{1-4}$.

O interesse pelo papel dos aspectos temporais auditivos foi originado pela constatação de sua atribuição fundamental na habilidade da comunicação humana. Os aspectos temporais auditivos estão relacionados intimamente na capacidade do indivíduo em reconhecer, discernir e perceber os aspectos segmentais e suprassegmentais da fala ${ }^{5,6}$. Além disso, sabe-se que a integridade desses aspectos é um pré-requisito para que o sistema auditivo defina a duração dos sons, o intervalo que os separa e a sequência em que ocorrem, habilidades essas, imprescindíveis para o processamento da música e da fala ${ }^{3,7,8}$.

Os aspectos temporais podem ser divididos em categorias que auxiliam no entendimento de alguns 
dos mecanismos e processos do sistema nervoso central. A ordenação e a resolução temporal, duas dessas categorias $^{6}$, de acordo com a literatura ${ }^{8,9}$ exercem funções essenciais na percepção da fala contínua e de suas partes isoladas, no aprendizado e na compreensão da linguagem, logo se constituem em condição para habilidades linguísticas, bem como na aquisição da leitura e escrita ${ }^{6}$.

A identificação das dificuldades escolares apresentadas pelos estudantes está aumentando. De acordo com os dados da UNESCO, "dos 41 países que compõem a região da América Latina e Caribe, o Brasil possui a maior taxa de repetência na educação básica: 18,7\%" ${ }^{10}$. Nessa educação básica, a maioria dos estudantes encontram-se na adolescência, que corresponde a fase de maior vulnerabilidade do indivíduo, determinada pelo processo de crescimento e desenvolvimento, colocando-o frágil nas mais diferentes situações, como as dificuldades escolares, por exemplo. Portanto, lidar com o insucesso escolar, baixo rendimento e as implicações para auto-avaliação do estudante, familiares e professores aponta para necessidade da busca de alternativas que minimizem tal situação.

Dessa forma, o presente estudo pretende contribuir para esclarecer a relação entre desempenho escolar e aspectos temporais auditivos, visando a ampliação da literatura acerca da avaliação do processamento temporal em adolescentes com dificuldades escolares. A faixa etária referida foi escoIhida uma vez que, instrumentos para avaliação das habilidades auditivas são dependentes da função neural e devem ser interpretados dentro de um contexto neuromaturacional. Além disso a literatura $^{11}$ acredita que por volta dos 11 a 12 anos, início da adolescência, o desempenho nos testes comportamentais auditivos centrais alcancem valores obtidos em adultos.

Sendo assim, o objetivo desse estudo foi investigar a relação entre aspectos temporais auditivos e desenvolvimento escolar em adolescentes do $6^{\circ}$ ano do Ensino Fundamental.

\section{MÉTODO}

Trata-se de estudo descritivo-transversal com amostra de conveniência, composta por 82 estudantes do 6 o ano de uma escola de financiamento privado na Região Metropolitana de Belo Horizonte. A faixa etária dos sujeitos variou de 11 anos e dois meses a 13 anos e dois meses, sendo 44 do sexo feminino e 38 do sexo masculino. Vale ressaltar que a amostra inicial era composta por 85 estudantes, porém foram excluídos três escolares da pesquisa, uma vez que após o período de férias os mesmos não retornaram, porque trocaram de escola.

Após análise e aprovação da pesquisa pela coordenação da escola, obteve-se autorização para realização dos procedimentos na própria escola de origem da amostra.

Foram considerados como critérios de inclusão para constituir o grupo de estudo, os estudantes sem evidências ou histórico de alterações cognitivas, neurológicas ou motoras que concordaram em participar da pesquisa e tiveram o Termo Livre e Esclarecido assinado por eles próprios e pelos responsáveis.

Foram excluídos da pesquisa aqueles que não conseguiram realizar os testes propostos, desistiram durante a aplicação das avaliações, apresentaram diagnóstico prévio de alteração auditiva, não responderam adequadamente ao formulário e apresentaram histórico de alterações cognitivas, neurológicas ou motoras.

Na primeira etapa da avaliação, todos os indivíduos foram submetidos a um formulário da caracterização da amostra, composto por nove perguntas, elaborado pelas pesquisadoras (Figura 1). O formulário foi preenchido pelos sujeitos de pesquisa em uma sala de aula com duração aproximada de 10 minutos, cujo objetivo foi conhecer o perfil dos adolescentes que fizeram parte da pesquisa, além da percepção dos mesmos sobre suas possíveis dificuldades dentro da sala de aula.

Ainda na primeira etapa foi aplicado o Teste de Desempenho Escolar (TDE) em todos os sujeitos da pesquisa. O TDE é composto por três subtestes: Escrita, Aritmética e Leitura. Cada um dos subtestes apresenta uma escala de itens em ordem de dificuldade, que foram propostos ao estudante. O teste é apresentado sob forma de um caderno contendo os três subtestes. A aplicação foi realizada em grupo de quatro estudantes, com duração máxima de 40 minutos, sempre iniciando pelo subteste de Escrita, seguido pelo de Aritmética e por fim, o subteste de Leitura. O padrão utilizado como critério de referência para classificar as respostas dos estudantes foi a padronização indicada no Manual de Aplicação e Interpretação do TDE ${ }^{12}$.

Cabe ressaltar que o resultado do Teste de Desempenho Escolar supracitado foi utilizado para a distribuição da amostra em três grupos de análise de acordo com a classificação do TDE, a saber:

- Grupo 1: indivíduos com desempenho inferior,

- Grupo 2: indivíduos com desempenho médio,

- Grupo 3: indivíduos com desempenho superior.

A segunda etapa da avaliação foi composta pelos testes que avaliam o Processamento Auditivo. Essa etapa iniciou-se pela aplicação individual 


\section{Formulário de CARActerização da Amostra \\ O formulário fol elaborado por Stela Maris AgUiar lemos e SUlamita da Silva Marcelino Terto}

CÓDIGO:

SEXo: ( ) Masculino ( ) Feminino IDADE:

PREFERÊNCIA MANUAL:

SÉRIE:

1) VOCÊ TEM DIFICULDADE EM OUVIR?

( ) SIM

( ) NÃO

2) VoCÊ ESCUTA BEM EM AMBIENTE RUIDOSO (BARULHENTO)?

( ) SIM

( ) NÃO

3) VocÊ ESCUtA BEM EM AMBIENTE SILENCIOSO?

( ) SIM

( ) NÃO

4) VOCÊ JÁ REPETIU ALGUMA SÉRIE?

( ) SIM

( ) NÃO

QUANTAS VEZES?

EM QUE SÉRIE?

5) MARQUE O GRAU DE DIFICULDADE QUE VOCÊ APRESENTA NAS SEGUINTES HABILIDADES:

- FALA

NENHUMA $0 \_$___ $2 \_3 \_4 \_5 \_6 \_7 \_8 \_9 \_10$ MUITA

- $\quad$ Leitura

NeNHUMA $0 \_$___ $2 \_3 \_4 \_5 \_6 \_7 \_8 \_9 \_10$ MUITA

- ESCRITA

NENHUMA $0 \_$_ $1 \_2 \_3 \_4 \_5 \_6 \_7 \_$_ $8 \_9 \_10$ MUITA

6) EM RELAÇÃO AO DESEMPENHO ESCOLAR, VOCÊ:

( ) ESTÁ ABAIXO DA MÉDIA EM MAIS DE UMA DISCIPLINA.

( ) ESTÁ NA MÉDIA OU ACIMA.

7) VOCÊ TOMA ALGUM MEDICAMENTO?

( ) SIM

( ) NÃO

QUAL O MOTIVO?

8) VOCÊ FAZ OU JÁ ALGUM TRATAMENTO OU ACOMPANHAMENTO COM FONOAUDIÓLOGO?

( ) SIM

( ) NÃO

9) QUAIS DAS ATIVIDADES EXTRA-CURRICULARES VOCÊ REALIZA OU JÁ REALIZOU?

( ) DANÇA

( ) LÍNGUA ESTRANGEIRA

QUAL (IS)?

( ) INFORMÁTICA

( ) ESPORTE

QUAL (IS)?

( ) AULAS DE MÚSICA OU DE INSTRUMENTO MUSICAL

QUAL (IS)?

Figura 1 - Formulário de caracterização da amostra. 
dos Testes de Padrão Tonal de Duração (TPD) e Padrão Tonal de Frequência (TPF) propostos pela literatura ${ }^{13}$, utilizando-se como referência estudo com escolares ${ }^{14}$. Ambos os testes foram apresentados por meio de uma gravação (estímulos sonoros do tipo flauta) em CD, utilizando-se um netbook acoplado em um fone TDH-39 (padrão ANSI, 1969), com intensidade fixa de 65 dBNA aferida por meio de um decibelímetro. Tais cuidados visavam à padronização da apresentação do estímulo.

No Teste de Padrão Tonal de Frequência (TPF) foram apresentadas dez sequências compostas por três estímulos e dez sequências compostas por quatro estímulos com combinações de frequência baixa $(440 \mathrm{~Hz})$ e alta $(493 \mathrm{~Hz})$ com duração fixa. Já o Teste de Padrão Tonal de Duração (TPD) seguiu procedimento semelhante ao TPF, porém com diferenças quanto à duração do estímulo sonoro, sendo constituído de dez tons de duração longa (161ms) e dez tons de duração curta (59ms), ambas com frequência fixa. A avaliação dos testes foi realizada individualmente. Cada teste foi realizado duas vezes, no qual o estudante recebia uma folha de resposta no início da aplicação dos testes e era instruído em um primeiro momento marcar suas respostas com símbolos. Os símbolos adotados para os estímulos longo e curto no TPD eram representados por um traço (__ ) e um asterisco $\left(^{*}\right)$ respectivamente, e os símbolos adotados para os estímulos alto e baixo no TPF eram representados por um xis $(X)$ e um círculo $(O)$ respectivamente. A segunda marcação das respostas dos testes era realizada pela aplicadora, por meio da nomeação dos estímulos pelo estudante. Nessa, o adolescente expressava oralmente sua resposta (curto ou longo) no TPD ou (alto ou baixo) no TPF e a aplicadora anotava na mesma folha de resposta utilizada anteriormente pelo adolescente as iniciais do estímulo (C para estímulo curto e $L$ para estímulo longo) no TPD ou (A para estímulo alto e B para estímulo baixo) no TPF.

Vale ressaltar que, neste estudo, os estímulos do TPF e TPD foram aplicados binauralmente, tendo em vista que estudos na literatura não relataram diferença com significância estatística quando comparados os desempenhos das orelhas esquerda e direita, tanto para o Teste Padrão de Frequência (TPF), quanto para o Teste Padrão de Duração (TPD). De acordo com a literatura ${ }^{15}$, tais podem ser aplicados também em campo livre. Como critério de análise, nesse estudo foram utilizados os valores propostos pela literatura ${ }^{16}$.

Para terminar a segunda e última etapa da avaliação, foi realizado o Teste GIN, proposto pela literatura ${ }^{17}$, utilizando-se com referência a literatura nacional ${ }^{18}$.
Nesse teste, os estímulos do tipo white noise estão distribuídos em quatro faixas-testes e uma faixa-treino. Consiste em estímulos de seis segundos de ruído branco intercalados com intervalos de silêncio (gaps). Os gaps são aleatórios e possuem durações variadas $(2,3,4,5,6,8,10,12$, 15 e 20ms). Os estímulos poderão conter número de gaps diferentes: um, dois, três ou nenhum. A aplicação do teste, na presente pesquisa, utilizou somente a faixa-treino e uma faixa-teste. Os estímulos foram apresentados na intensidade de 60 dBNA, em uma gravação do teste em $C D$, utilizando-se um equipamento PAC-2000 da marca Acústica Orlandi conectado a um CD Player e fones TDH-39 (padrão ANSI, 1969).

Antes de se iniciar a aplicação da faixa-teste, durante a execução da faixa-treino, os estudantes foram instruídos a levantar o dedo indicador ao escutarem os gaps embutidos no ruído. A orientação para execução do teste foi repetida quando o adolescente não compreendeu a tarefa. A aplicadora anotou em uma folha de registro as respostas presentes utilizando um círculo $(\mathrm{O})$, as respostas ausentes utilizando um traço (__ $)$ e os falso-positivos utilizando um asterisco $\left(^{\star}\right)$. Os falso-positivos consistiam em respostas dadas pelos estudantes na ausência de gaps. É importante ressaltar que, cada adolescente poderia apresentar no máximo duas respostas falso-positivas. A partir de então as mesmas foram consideradas como respostas ausentes, sendo descontadas do total de acertos na realização do cálculo do teste.

A distribuição da amostra ocorreu baseada no desempenho de cada indivíduo no Teste de Desempenho Escolar. Levou-se em consideração a pontuação obtida em cada escore dos subtestes Escrita, Aritmética e Leitura. A classificação dos grupos foi estimada somando-se os escores de todos os subtestes e os indivíduos foram assim distribuídos: Grupo I: correspondem aos estudantes que obtiveram desempenho superior, $54,3 \%(\mathrm{~N}=44)$ da amostra total; Grupo II: correspondem aos estudantes que obtiveram desempenho médio, 38,3\% ( $N=31)$ da amostra total e Grupo III: correspondem aos estudantes que obtiveram desempenho inferior, 7,4\% $(\mathrm{N}=6)$ da amostra total. O desempenho dos Grupos I, II e III foram comparados quanto a realização dos testes Padrão Tonal de Frequência, Padrão Tonal de Duração e Teste GIN.

Essa pesquisa foi aprovada pelo COEP da Universidade Federal de Minas Gerais sob o parecer $n^{\circ}$ ETIC 590/09.

As informações coletadas foram digitadas em um banco de dados desenvolvido no Excel $囚$. Os resultados descritivos foram obtidos utilizando frequências e porcentagens para as características 
das diversas variáveis categóricas e da obtenção de medidas de tendência central (média e mediana) e medidas de dispersão (desvio-padrão) para as quantitativas.

Para verificar a associação entre as variáveis categóricas utilizou-se o teste Qui-Quadrado de Pearson. Para utilização deste teste era necessário que pelo menos $80 \%$ das caselas não tivessem valor esperado menor que 5. Caso esta condição não fosse satisfeita utilizou-se o teste Exato de Fisher. No caso em que uma das duas variáveis tinha categorias ordenáveis utilizou-se o teste Qui-quadrado de tendência, que é sensível à tendência linear entre as variáveis estudadas. Para a comparação das variáveis contínuas ou discretas (Grau de desempenho) utilizou o teste não paramétrico de Kruskal Wallis (equivalente à análise de variância para um fator), uma vez que há mais de dois grupos de comparação. Foram consideradas associações estatisticamente significantes, os resultados que apresentaram um nível de significância de 95\% (p-valor $\leq 0,05)$.

Para o processamento e análise dos dados foi utilizado o programa SPSS versão 12.

\section{RESULTADOS}

$\mathrm{Na}$ análise dos dados, observa-se que a idade dos escolares variou entre 11 e 13 anos, com uma média de 11,1 anos e desvio padrão de 0,4 anos. Observa-se um maior percentual de adolescentes do sexo feminino $53,1 \%(\mathrm{~N}=43)$, em comparação com percentual do sexo masculino $46,9 \%(\mathrm{~N}=38)$. No que se refere à auto-percepção da audição, foi observado que $14,8 \%(\mathrm{~N}=12)$ dos escolares pesquisados referiram possuir dificuldade em ouvir. Em relação ao uso de medicamentos, 23,5\% ( $\mathrm{N}=19)$ relataram utilizar constantemente algum remédio, a causa mais recorrente foi tratamento para alergia. Os resultados referentes aos testes que avaliaram o desempenho escolar e o processamento auditivo, juntamente com as respostas do questionário de caracterização da amostra encontram-se nas tabelas 1 a 5 .

Durante a análise dos dados, pode-se observar que, a média dos limiares de gap foi de $4,87 \mathrm{~ms}$, enquanto a média das porcentagens de acertos de gap foi de $71,86 \%$ para a amostra total.
A tabela 1 mostra os resultados do teste GIN por grupos do TDE e a correlação com as questões do formulário.

Destaca-se que todos os testes não sofreram influência com significância estatística nos itens "Escuta bem em ambiente ruidoso ou silencioso" e "Toma algum medicamento".

Foram observados que os valores no Teste Padrão de Duração no tipo de resposta "símbolo" variaram entre 7 e 10 acertos para 3 sons. Já no tipo de resposta "nomeação", os valores variaram entre 4 e 10 acertos. O valor da mediana foi 10 tanto para o tipo de resposta "nomeação" quanto para o "símbolo".

A tabela 2 mostra os resultados do teste Padrão de Duração com 3 Sons (TPDS3) por grupos do TDE e a correlação com as questões do formulário.

No mesmo teste, porém com 4 sons, observou-se que os valores no tipo de resposta "símbolo" variaram entre 6 e 10 acertos. Enquanto que no tipo de resposta "nomeação", os valores variaram entre 3 e 10 acertos. Os valores da mediana foram 10 e 9 para o tipo de resposta "símbolo" e para a "nomeação", respectivamente.

A tabela 3 mostra os resultados do teste Padrão de Duração com 4 Sons (TPDS4) por grupos do TDE e a correlação com as questões do formulário.

Os valores no Teste Padrão de Frequência tanto no tipo de resposta "símbolo", quanto no tipo de resposta "nomeação" variaram entre 0 e 10 acertos para 3 sons. $O$ valor da mediana encontrado para ambos os tipos de resposta foi de 5 .

A tabela 4 mostra os resultados do teste Padrão de Duração com 3 Sons (TPFS3) por grupos do TDE e a correlação com as questões do formulário.

Nos valores no Teste Padrão de Frequência para 4 sons também foram encontrados resultados semelhantes aos estímulos com 3 sons, uma vez que tanto no tipo de resposta "símbolo", quanto no tipo de resposta "nomeação" o número de acertos variou entre 0 e 10 e os valores da mediana foram 6 e 4 para o tipo de resposta "símbolo" e para a "nomeação", respectivamente.

A tabela 5 mostra os resultados do teste Padrão de Duração com 4 Sons (TPFS4) por grupos do TDE e a correlação com as questões do formulário. 
Tabela 1 - Resultado da associação entre o desempenho no Teste GIN relacionado à classificação no Teste de Desempenho Escolar e a resposta das questões do formulário de caracterização da amostra

\begin{tabular}{|c|c|c|c|c|c|}
\hline \multirow{2}{*}{ GIN (Gaps in noise) } & \multicolumn{2}{|c|}{ Normal } & \multicolumn{2}{|c|}{ Alterado } & \multirow{2}{*}{ valor-p } \\
\hline & $\mathbf{n}$ & $\%$ & $\mathbf{n}$ & $\%$ & \\
\hline \multicolumn{6}{|l|}{ Teste de Desempenho Escolar - Escrita } \\
\hline Superior & 34 & 89,5 & 4 & 10,5 & $0,632^{1}$ \\
\hline Médio & 23 & 82,1 & 5 & 17,9 & \\
\hline Inferior & 13 & 86,7 & 2 & 13,3 & \\
\hline \multicolumn{6}{|l|}{ Teste de Desempenho Escolar - Aritmética } \\
\hline Superior & 40 & 88,9 & 5 & 11,1 & $0,470^{1}$ \\
\hline Médio & 16 & 84,2 & 3 & 15,8 & \\
\hline Inferior & 14 & 82,4 & 3 & 17,6 & \\
\hline \multicolumn{6}{|l|}{ Teste de Desempenho Escolar - Leitura } \\
\hline Superior & 57 & 86,4 & 9 & 13,6 & $0,450^{1}$ \\
\hline Médio & 11 & 84,6 & 2 & 15,4 & \\
\hline Inferior & 2 & 100,0 & 0 & 0,0 & \\
\hline \multicolumn{6}{|l|}{ Teste de Desempenho Escolar - TDE } \\
\hline Superior & 39 & 88,6 & 5 & 11,4 & $0,553^{1}$ \\
\hline Médio & 26 & 83,9 & 5 & 16,1 & \\
\hline Inferior & 5 & 83,3 & 1 & 16,7 & \\
\hline \multicolumn{6}{|l|}{ Preferência manual } \\
\hline Direita & 39 & 90,7 & 4 & 9,3 & $0,332^{2}$ \\
\hline Esquerda & 31 & 81,6 & 7 & 18,4 & \\
\hline \multicolumn{6}{|l|}{ Sexo } \\
\hline Feminino & 59 & 85,5 & 10 & 14,5 & $1,000^{2}$ \\
\hline Masculino & 11 & 91,7 & 1 & 8,3 & \\
\hline \multicolumn{6}{|l|}{ Dificuldade em ouvir } \\
\hline Sim & 10 & 83,3 & 2 & 16,7 & $0,663^{2}$ \\
\hline Não & 60 & 87,0 & 9 & 13,0 & \\
\hline \multicolumn{6}{|l|}{ Repetiu alguma série } \\
\hline Sim & 8 & 72,7 & 3 & 27,3 & $0,167^{2}$ \\
\hline Não & 62 & 88,6 & 8 & 11,4 & \\
\hline \multicolumn{6}{|l|}{ Desempenho escolar } \\
\hline Abaixo da média & 14 & 87,5 & 2 & 12,5 & $1,000^{2}$ \\
\hline Média ou acima da média & 56 & 86,2 & 9 & 13,8 & \\
\hline \multicolumn{6}{|l|}{ Acompanhamento com Fonoaudiólogo } \\
\hline $\operatorname{Sim}$ & 3 & 42,9 & 4 & 57,1 & $0,006^{2}$ \\
\hline Não & 67 & 90,5 & 7 & 9,5 & \\
\hline \multicolumn{6}{|l|}{ Atividade de Língua estrangeira } \\
\hline Nenhum & 32 & 82,1 & 7 & 17,9 & $0,339^{2}$ \\
\hline Língua estrangeira & 38 & 90,5 & 4 & 9,5 & \\
\hline \multicolumn{6}{|l|}{ Atividade de Musicalização } \\
\hline Nenhum & 46 & 82,1 & 10 & 17,9 & $0,159^{2}$ \\
\hline Musicalização & 24 & 96,0 & 1 & 4,0 & \\
\hline
\end{tabular}

Legenda: ${ }^{1}$ Teste Qui-quadrado de tendência; ${ }^{2}$ Teste exato de Fisher. 
Tabela 2 - Resultado da associação entre o desempenho nos testes padrão de duração com 3 sons relacionado à classificação no teste de desempenho escolar e a resposta das questões do formulário de caracterização da amostra

\begin{tabular}{|c|c|c|c|c|c|c|c|c|c|c|c|c|}
\hline \multirow{3}{*}{$\begin{array}{l}\text { Variável Testes } \\
\text { TDE - Escrita }\end{array}$} & \multicolumn{4}{|c|}{ TPDS3 } & \multirow{2}{*}{\multicolumn{2}{|c|}{ Valor - $p$}} & \multicolumn{4}{|c|}{ TPDN3 } & \multirow{2}{*}{\multicolumn{2}{|c|}{ Valor - $p$}} \\
\hline & \multicolumn{2}{|c|}{ Normal } & \multicolumn{2}{|c|}{ Alterado } & & & \multicolumn{2}{|c|}{ Normal } & \multicolumn{2}{|c|}{ Alterado } & & \\
\hline & $\mathbf{n}$ & $\%$ & $\mathbf{n}$ & $\%$ & & & $\mathbf{n}$ & $\%$ & $\mathbf{n}$ & $\%$ & & \\
\hline Superior & 32 & 84,2 & 6 & 15,8 & $0,311^{1}$ & & 22 & 57,9 & 16 & 42,1 & & \\
\hline Médio & 21 & 75,0 & 7 & 25,0 & & & 18 & 64,3 & 10 & 35,7 & & \\
\hline Inferior & 11 & 73,3 & 4 & 26,7 & & & 9 & 60,0 & 6 & 40,0 & & \\
\hline \multicolumn{13}{|l|}{$\begin{array}{c}\text { TDE - } \\
\text { Aritmética }\end{array}$} \\
\hline Superior (1) & 42 & 93,3 & 3 & 6,7 & $0,008^{1}$ & $\mathbf{0 , 0 3 0}(1 \times 3)$ & 30 & 66,7 & 15 & 33,3 & & \\
\hline Médio (2) & 10 & 52,6 & 9 & 47,4 & & $0,270(2 \times 3)$ & 9 & 47,4 & 10 & 52,6 & & \\
\hline Inferior (3) & 12 & 70,6 & 5 & 29,4 & & $\mathbf{0 , 0 0 1}(1 \times 2)$ & 10 & 58,8 & 7 & 41,2 & & \\
\hline \multicolumn{13}{|l|}{ TDE - Leitura } \\
\hline Superior & 53 & 80,3 & 13 & 19,7 & $0,962^{1}$ & & 44 & 66,7 & 22 & 33,3 & $0,037^{1}$ & $1,000(1 \times 3)$ \\
\hline Médio & 10 & 76,9 & 3 & 23,1 & & & 4 & 30,8 & 9 & 69,2 & & $1,000(2 \times 3)$ \\
\hline Inferior & 1 & 50,0 & 1 & 50,0 & & & 1 & 50,0 & 1 & 50,0 & & $\mathbf{0 , 0 3 4}(1 \times 2)$ \\
\hline \multicolumn{13}{|l|}{ TDE } \\
\hline Superior (1) & 39 & 88,6 & 5 & 11,4 & $0,010^{1}$ & $\mathbf{0 , 0 4 4}(1 \times 3)$ & 28 & 63,6 & 16 & 36,4 & & \\
\hline Médio (2) & 22 & 71,0 & 9 & 29,0 & & $0,367(2 \times 3)$ & 18 & 58,1 & 13 & 41,9 & & \\
\hline Inferior (3) & 3 & 50,0 & 3 & 50,0 & & $0,053(1 \times 2)$ & 3 & 50,0 & 3 & 50,0 & & \\
\hline \multicolumn{13}{|l|}{$\begin{array}{l}\text { Preferência } \\
\text { manual }\end{array}$} \\
\hline Direita & 56 & 81,2 & 13 & 18,8 & $0,265^{2}$ & & 44 & 63,8 & 25 & 36,2 & & \\
\hline Esquerda & 8 & 66,7 & 4 & 33,3 & & & 5 & 41,7 & 7 & 58,3 & & \\
\hline \multicolumn{13}{|l|}{ Sexo } \\
\hline Feminino & 36 & 83,7 & 7 & 16,3 & $0,289^{2}$ & & 28 & 65,1 & 15 & 34,9 & & \\
\hline Masculino & 28 & 73,7 & 10 & 26,3 & & & 21 & 55,3 & 17 & 44,7 & & \\
\hline \multicolumn{13}{|l|}{$\begin{array}{c}\text { Dificuldade em } \\
\text { ouvir }\end{array}$} \\
\hline Sim & 12 & 100 & 0 & 0,0 & $0,062^{2}$ & & 8 & 66,7 & 4 & 33,3 & & \\
\hline Não & 52 & 75,4 & 17 & 24,6 & & & 41 & 59,4 & 28 & 40,6 & & \\
\hline \multicolumn{13}{|l|}{$\begin{array}{c}\text { Repetiu alguma } \\
\text { série }\end{array}$} \\
\hline Sim & 7 & 63,6 & 4 & 36,4 & $0,230^{2}$ & & 4 & 36,4 & 7 & 63,6 & & \\
\hline Não & 57 & 81,4 & 13 & 18,6 & & & 45 & 64,3 & 25 & 35,7 & & \\
\hline \multicolumn{13}{|l|}{$\begin{array}{l}\text { Desempenho } \\
\text { escolar }\end{array}$} \\
\hline Abaixo da média & 12 & 75,0 & 4 & 25,0 & $0,734^{2}$ & & 8 & 50,0 & 8 & 50,0 & & \\
\hline $\begin{array}{c}\text { Média ou acima } \\
\text { da média }\end{array}$ & 52 & 80,0 & 13 & 20,0 & & & 41 & 63,1 & 24 & 36,9 & & \\
\hline \multicolumn{13}{|l|}{ Fonoaudiólogo } \\
\hline Sim & 6 & 85,7 & 1 & 14,3 & $1,000^{2}$ & & 4 & 57,1 & 3 & 42,9 & & \\
\hline Não & 58 & 78,4 & 16 & 21,6 & & & 45 & 60,8 & 29 & 39,2 & & \\
\hline \multicolumn{13}{|l|}{ L. estrangeira } \\
\hline Nenhum & 31 & 79,5 & 8 & 20,5 & $1,000^{2}$ & & 22 & 56,4 & 17 & 43,6 & & \\
\hline $\begin{array}{c}\text { Língua } \\
\text { estrangeira }\end{array}$ & 33 & 78,6 & 9 & 21,4 & & & 27 & 64,3 & 15 & 35,7 & & \\
\hline \multicolumn{13}{|l|}{ Musicalização } \\
\hline Nenhum & 42 & 75,0 & 14 & 25,0 & $0,244^{2}$ & & 34 & 60,7 & 22 & 39,3 & & \\
\hline Musicalização & 22 & 88,0 & 3 & 12,0 & & & 15 & 60,0 & 10 & 40,0 & & \\
\hline
\end{tabular}

Legenda: TPDS3: Teste Padrão de Duração com 3 Sons (Símbolo) ; TPDN3: Teste Padrão de Duração com 3 Sons (Nomeação); TDE: Teste de Desempenho Escolar; L. estrangeira: Língua estrangeira .

1 Teste Qui-quadrado de tendência; ${ }^{2}$ Teste exato de Fisher. 
Tabela 3 - Resultado da associação entre o desempenho nos testes padrão de duração com 4 sons relacionado à classificação no teste de desempenho escolar e a resposta das questões do formulário de caracterização da amostra

\begin{tabular}{|c|c|c|c|c|c|c|c|c|c|c|c|}
\hline \multirow{3}{*}{$\begin{array}{l}\text { Variável } \\
\text { TDE - Escrita }\end{array}$} & \multicolumn{4}{|c|}{ TPDS4 } & \multirow{3}{*}{ Valor - $p$} & \multicolumn{4}{|c|}{ TPDN4 } & \multirow{2}{*}{\multicolumn{2}{|c|}{ Valor - $p$}} \\
\hline & \multicolumn{2}{|c|}{ Normal } & \multicolumn{2}{|c|}{ Alterado } & & \multicolumn{2}{|c|}{ Normal } & \multicolumn{2}{|c|}{ Alterado } & & \\
\hline & $\mathbf{n}$ & $\%$ & $\mathbf{n}$ & $\%$ & & $\mathbf{n}$ & $\%$ & $\mathbf{n}$ & $\%$ & & \\
\hline Superior & 36 & 94,7 & 2 & 5,3 & $0,236^{1}$ & 32 & 84,2 & 6 & 15,8 & $0,311^{1}$ & \\
\hline Médio & 23 & 82,1 & 5 & 17,9 & & 21 & 75,0 & 7 & 25,0 & & \\
\hline Inferior & 13 & 86,7 & 2 & 13,3 & & 11 & 73,3 & 4 & 26,7 & & \\
\hline \multicolumn{12}{|l|}{ TDE - Aritmética } \\
\hline Superior (1) & 41 & 91,1 & 4 & 8,9 & $0,356^{1}$ & 39 & 86,7 & 6 & 13,3 & $0,332^{1}$ & \\
\hline Médio (2) & 17 & 89,5 & 2 & 10,5 & & 11 & 57,9 & 8 & 42,1 & & \\
\hline Inferior (3) & 14 & 82,4 & 3 & 17,6 & & 14 & 82,4 & 3 & 17,6 & & \\
\hline \multicolumn{12}{|l|}{ TDE - Leitura } \\
\hline Superior & 59 & 89,4 & 7 & 10,6 & $0,260^{1}$ & 55 & 83,3 & 11 & 16,7 & $0,045^{1}$ & $0,324(1 \times 3)$ \\
\hline Médio & 11 & 84,6 & 2 & 15,4 & & 8 & 61,5 & 5 & 38,5 & & $1,000(2 \times 3)$ \\
\hline Inferior & 2 & 100,0 & 0 & 0,0 & & 1 & 50,0 & 1 & 50,0 & & $\mathbf{0 , 0 1 2}(1 \times 2)$ \\
\hline \multicolumn{12}{|l|}{ TDE } \\
\hline Superior (1) & 40 & 90,9 & 4 & 9,1 & $0,215^{1}$ & 36 & 81,8 & 8 & 18,2 & $0,395^{1}$ & \\
\hline Médio (2) & 28 & 90,3 & 3 & 9,7 & & 24 & 77,4 & 7 & 22,6 & & \\
\hline Inferior (3) & 4 & 66,7 & 2 & 33,3 & & 4 & 66,7 & 2 & 33,3 & & \\
\hline \multicolumn{12}{|l|}{$\begin{array}{c}\text { Preferência } \\
\text { manual }\end{array}$} \\
\hline Direita & 63 & 91,3 & 6 & 8,7 & $0,125^{2}$ & 55 & 79,7 & 14 & 20,3 & $0,709^{2}$ & \\
\hline Esquerda & 9 & 75,0 & 3 & 25,0 & & 9 & 75,0 & 3 & 25,0 & & \\
\hline \multicolumn{12}{|l|}{ Sexo } \\
\hline Feminino & 39 & 90,7 & 4 & 9,3 & $0,728^{2}$ & 35 & 81,4 & 8 & 18,6 & $0,597^{2}$ & \\
\hline Masculino & 33 & 86,8 & 5 & 13,2 & & 29 & 76,3 & 9 & 23,7 & & \\
\hline \multicolumn{12}{|l|}{$\begin{array}{c}\text { Dificuldade em } \\
\text { ouvir }\end{array}$} \\
\hline Sim & 11 & 91,7 & 1 & 8,3 & $1,000^{2}$ & 11 & 91,7 & 1 & 8,3 & $0,444^{2}$ & \\
\hline Não & 61 & 88,4 & 8 & 11,6 & & 53 & 76,8 & 16 & 23,2 & & \\
\hline \multicolumn{12}{|l|}{$\begin{array}{c}\text { Repetiu alguma } \\
\text { série }\end{array}$} \\
\hline Sim & 9 & 81,8 & 2 & 18,2 & $0,602^{2}$ & 6 & 54,5 & 5 & 45,5 & $0,047^{2}$ & \\
\hline Não & 63 & 90,0 & 7 & 10,0 & & 58 & 82,9 & 12 & 17,1 & & \\
\hline \multicolumn{12}{|l|}{$\begin{array}{l}\text { Desempenho } \\
\text { escolar }\end{array}$} \\
\hline \multirow{2}{*}{$\begin{array}{l}\text { Abaixo da média } \\
\text { Média ou acima da } \\
\text { média }\end{array}$} & 14 & 87,5 & 2 & 12,5 & $1,000^{2}$ & 14 & 87,5 & 2 & 12,5 & $0,501^{2}$ & \\
\hline & 58 & 89,2 & 7 & 10,8 & & 50 & 76,9 & 15 & 23,1 & & \\
\hline \multicolumn{12}{|l|}{ Fonoaudiólogo } \\
\hline Sim & 6 & 85,7 & 1 & 14,3 & $0,576^{2}$ & 5 & 71,4 & 2 & 28,6 & $0,633^{2}$ & \\
\hline Não & 66 & 89,2 & 8 & 10,8 & & 59 & 79,7 & 15 & 20,3 & & \\
\hline \multicolumn{12}{|l|}{ L.estrangeira } \\
\hline Nenhum & 37 & 94,9 & 2 & 5,1 & $0,157^{2}$ & 32 & 82,1 & 7 & 17,9 & $0,592^{2}$ & \\
\hline Língua estrangeira & 35 & 83,3 & 7 & 16,7 & & 32 & 76,2 & 10 & 23,8 & & \\
\hline \multicolumn{12}{|l|}{ Musicalização } \\
\hline Nenhum & 49 & 87,5 & 7 & 12,5 & $0,714^{2}$ & 42 & 75,0 & 14 & 25,0 & $0,244^{2}$ & \\
\hline Musicalização & 23 & 92,0 & 2 & 8,0 & & 22 & 88,0 & 3 & 12,0 & & \\
\hline
\end{tabular}

Legenda: TPDS4: Teste Padrão de Duração com 4 Sons (Símbolo) ; TPDN4: Teste Padrão de Duração com 4 Sons

(Nomeação); TDE: Teste de Desempenho Escolar; L. estrangeira: Língua estrangeira .

${ }^{1}$ Teste Qui-quadrado de tendência; ${ }^{2}$ Teste exato de Fisher. 
Tabela 4 - Resultado da associação entre o desempenho nos testes padrão de frequência com 3 sons relacionado à classificação no teste de desempenho escolar e a resposta das questões do formulário de caracterização da amostra

\begin{tabular}{|c|c|c|c|c|c|c|c|c|c|c|c|}
\hline \multirow{3}{*}{$\begin{array}{c}\text { Variável } \\
\text { TDE - Escrita }\end{array}$} & \multicolumn{4}{|c|}{ TPFS3 } & \multirow{3}{*}{ Valor - p } & \multicolumn{4}{|c|}{ TPFN3 } & \multirow{2}{*}{\multicolumn{2}{|c|}{ Valor - $p$}} \\
\hline & \multicolumn{2}{|c|}{ Normal } & \multicolumn{2}{|c|}{ Alterado } & & \multicolumn{2}{|c|}{ Normal } & \multicolumn{2}{|c|}{ Alterado } & & \\
\hline & $\mathbf{n}$ & $\%$ & $\mathbf{n}$ & $\%$ & & $\mathbf{n}$ & $\%$ & $\mathbf{n}$ & $\%$ & & \\
\hline Superior & 20 & 52,6 & 18 & 47,4 & $0,061^{1}$ & 16 & 42,1 & 22 & 57,9 & $0,050^{1}$ & $\mathbf{0 , 0 4 6}(1 \times 3)$ \\
\hline Médio & 10 & 35,7 & 18 & 64,3 & & 9 & 32,1 & 19 & 67,9 & & $0,276(2 \times 3)$ \\
\hline Inferior & 4 & 26,7 & 11 & 73,3 & & 2 & 13,3 & 13 & 86,7 & & $0,409(1 \times 2)$ \\
\hline \multicolumn{12}{|l|}{ TDE - Aritmética } \\
\hline Superior (1) & 20 & 44,4 & 25 & 55,6 & $0,532^{1}$ & 17 & 37,8 & 28 & 62,2 & $0,285^{1}$ & \\
\hline Médio (2) & 8 & 42,1 & 11 & 57,9 & & 6 & 31,6 & 13 & 68,4 & & \\
\hline Inferior (3) & 6 & 35,3 & 11 & 64,7 & & 4 & 23,5 & 13 & 76,5 & & \\
\hline \multicolumn{12}{|l|}{ TDE - Leitura } \\
\hline Superior & 29 & 43,9 & 37 & 56,1 & $0,584^{1}$ & 25 & 37,9 & 41 & 62,1 & $0,178^{1}$ & \\
\hline Médio & 4 & 30,8 & 9 & 69,2 & & 1 & 7,7 & 12 & 92,3 & & \\
\hline Inferior & 1 & 50,0 & 1 & 50,0 & & 1 & 50,0 & 1 & 50,0 & & \\
\hline \multicolumn{12}{|l|}{ TDE } \\
\hline Superior (1) & 22 & 50,0 & 22 & 50,0 & $0,073^{1}$ & 18 & 40,9 & 26 & 59,1 & $0,118^{1}$ & \\
\hline Médio (2) & 11 & 35,5 & 20 & 64,5 & & 9 & 29,0 & 22 & 71,0 & & \\
\hline Inferior (3) & 1 & 16,7 & 5 & 83,3 & & 0 & 0,0 & 6 & 100,0 & & \\
\hline \multicolumn{12}{|l|}{$\begin{array}{l}\text { Preferência } \\
\text { manual }\end{array}$} \\
\hline Direita & 31 & 44,9 & 38 & 55,1 & $0,225^{2}$ & 24 & 34,8 & 45 & 65,2 & $0,742^{2}$ & \\
\hline Esquerda & 31 & 44,9 & 38 & 55,1 & & 3 & 25,0 & 9 & 75,0 & & \\
\hline \multicolumn{12}{|l|}{ Sexo } \\
\hline Feminino & 17 & 39,5 & 26 & 60,5 & $0,659^{2}$ & 13 & 30,2 & 30 & 69,8 & $0,638^{2}$ & \\
\hline Masculino & 17 & 44,7 & 21 & 55,3 & & 14 & 36,8 & 24 & 63,2 & & \\
\hline \multicolumn{12}{|l|}{$\begin{array}{c}\text { Dificuldade em } \\
\text { ouvir }\end{array}$} \\
\hline Sim & 7 & 58,3 & 5 & 41,7 & $0,342^{2}$ & 4 & 33,3 & 8 & 66,7 & $1,000^{2}$ & \\
\hline Não & 27 & 39,1 & 42 & 60,9 & & 23 & 33,3 & 46 & 66,7 & & \\
\hline \multicolumn{12}{|l|}{$\begin{array}{c}\text { Repetiu alguma } \\
\text { série }\end{array}$} \\
\hline Sim & 2 & 18,2 & 9 & 81,8 & $0,108^{2}$ & 1 & 9,1 & 10 & 90,9 & $0,089^{2}$ & \\
\hline Não & 32 & 45,7 & 38 & 54,3 & & 26 & 37,1 & 44 & 62,9 & & \\
\hline \multicolumn{12}{|l|}{$\begin{array}{l}\text { Desempenho } \\
\text { escolar }\end{array}$} \\
\hline Abaixo da média & 5 & 31,3 & 11 & 68,8 & $0,405^{2}$ & 4 & 25,0 & 12 & 75,0 & $0,559^{2}$ & \\
\hline $\begin{array}{l}\text { Média ou acima da } \\
\text { média }\end{array}$ & 29 & 44,6 & 36 & 55,4 & & 23 & 35,4 & 42 & 64,6 & & \\
\hline \multicolumn{12}{|l|}{ Fonoaudiólogo } \\
\hline Sim & 3 & 42,9 & 4 & 57,1 & $1,000^{2}$ & 2 & 28,6 & 5 & 71,4 & $1,000^{2}$ & \\
\hline Não & 31 & 41,9 & 43 & 58,1 & & 25 & 33,8 & 49 & 66,2 & & \\
\hline \multicolumn{12}{|l|}{ L. estrangeira } \\
\hline Nenhum & 16 & 41,0 & 23 & 59,0 & $1,000^{2}$ & 12 & 30,8 & 27 & 69,2 & $0,814^{2}$ & \\
\hline Língua estrangeira & 18 & 42,9 & 24 & 57,1 & & 15 & 35,7 & 27 & 64,3 & & \\
\hline \multicolumn{12}{|l|}{ Musicalização } \\
\hline Nenhum & 22 & 39,3 & 34 & 60,7 & $0,477^{2}$ & 20 & 35,7 & 36 & 64,3 & $0,613^{2}$ & \\
\hline Musicalização & 12 & 48,0 & 13 & 52,0 & & 7 & 28,0 & 18 & 72,0 & & \\
\hline
\end{tabular}

Legenda: TPFS3: Teste Padrão de Frequência com 3 Sons (Símbolo); TPFN4: Teste Padrão de Frequência com 4 Sons (Nomeação); TDE: Teste de Desempenho Escolar; L. estrangeira: Língua estrangeira.

${ }^{1}$ Teste Qui-quadrado de tendência; ${ }^{2}$ Teste exato de Fisher. 
Tabela 5 - Resultado da associação entre o desempenho nos testes padrão de frequência com 4 sons relacionado à classificação no teste de desempenho escolar e a resposta das questões do formulário de caracterização da amostra

\begin{tabular}{|c|c|c|c|c|c|c|c|c|c|c|c|c|}
\hline \multirow{3}{*}{$\begin{array}{c}\text { Variável } \\
\text { TDE - Escrita }\end{array}$} & \multicolumn{4}{|c|}{ TPFS4 } & \multirow{2}{*}{\multicolumn{2}{|c|}{ Valor - p }} & \multicolumn{4}{|c|}{ TPFN4 } & \multirow{2}{*}{\multicolumn{2}{|c|}{ Valor - $p$}} \\
\hline & \multicolumn{2}{|c|}{ Normal } & \multicolumn{2}{|c|}{ Alterado } & & & \multicolumn{2}{|c|}{ Normal } & \multicolumn{2}{|c|}{ Alterado } & & \\
\hline & $n$ & $\%$ & $\mathbf{n}$ & $\%$ & & & $\mathbf{n}$ & $\%$ & $\mathrm{n}$ & $\%$ & & \\
\hline Superior & 23 & 60,5 & 15 & 39,5 & $\mathbf{0 , 0 4 3}$ & $\mathbf{0 , 0 4 7}(1 \times 3)$ & 20 & 52,6 & 18 & 47,4 & $0,046^{1}$ & $\mathbf{0 , 0 3 1}(1 \times 3)$ \\
\hline Médio & 11 & 39,3 & 17 & 60,7 & & $0,700(2 \times 3)$ & 4 & 14,3 & 24 & 85,7 & & $0,680(2 \times 3)$ \\
\hline Inferior & 5 & 33,3 & 10 & 66,7 & & $\mathbf{0 , 0 1 4}(1 \times 2)$ & 3 & 20,0 & 12 & 80,0 & & $\mathbf{0 , 0 0 1}(1 \times 2)$ \\
\hline \multicolumn{13}{|l|}{ TDE - Aritmética } \\
\hline Superior (1) & 23 & 51,1 & 22 & 48,9 & $0,333^{1}$ & & 19 & 42,2 & 26 & 57,8 & $0,052^{1}$ & \\
\hline Médio (2) & 10 & 52,6 & 9 & 47,4 & & & 5 & 26,3 & 14 & 73,7 & & \\
\hline Inferior (3) & 6 & 35,3 & 11 & 64,7 & & & 3 & 17,6 & 14 & 82,4 & & \\
\hline \multicolumn{13}{|l|}{ TDE - Leitura } \\
\hline Superior & 34 & 51,5 & 32 & 48,5 & $0,298^{1}$ & & 24 & 36,4 & 42 & 63,6 & $0,400^{1}$ & \\
\hline Médio & 4 & 30,8 & 9 & 69,2 & & & 2 & 15,4 & 11 & 84,6 & & \\
\hline Inferior & 1 & 50,0 & 1 & 50,0 & & & 1 & 50,0 & 1 & 50,0 & & \\
\hline \multicolumn{13}{|l|}{ TDE } \\
\hline Superior (1) & 25 & 56,8 & 19 & 43,2 & $0,099^{1}$ & & 20 & 45,5 & 24 & 54,5 & $0,019^{1}$ & $\mathbf{0 , 0 3 8}(1 \times 3)$ \\
\hline Médio (2) & 12 & 38,7 & 19 & 61,3 & & & 6 & 19,4 & 25 & 80,6 & & $1,000(2 \times 3)$ \\
\hline Inferior (3) & 2 & 33,3 & 4 & 66,7 & & & 1 & 16,7 & 5 & 83,3 & & $\mathbf{0 , 0 1 8}(1 \times 2)$ \\
\hline \multicolumn{13}{|l|}{$\begin{array}{c}\text { Preferência } \\
\text { manual }\end{array}$} \\
\hline Direita & 34 & 49,3 & 35 & 50,7 & $0,758^{2}$ & & 26 & 37,7 & 43 & 62,3 & $0,053^{2}$ & \\
\hline Esquerda & 5 & 41,7 & 7 & 58,3 & & & 1 & 8,3 & 11 & 91,7 & & \\
\hline \multicolumn{13}{|l|}{ Sexo } \\
\hline Feminino & 17 & 39,5 & 26 & 60,5 & $0,122^{2}$ & & 12 & 27,9 & 31 & 72,1 & $0,346^{2}$ & \\
\hline Masculino & 22 & 57,9 & 16 & 42,1 & & & 15 & 39,5 & 23 & 60,5 & & \\
\hline \multicolumn{13}{|l|}{$\begin{array}{c}\text { Dificuldade em } \\
\text { ouvir }\end{array}$} \\
\hline Sim & 7 & 58,3 & 5 & 41,7 & $0,538^{2}$ & & 8 & 66,7 & 4 & 33,3 & & \\
\hline Não & 32 & 46,4 & 37 & 53,6 & & & 41 & 59,4 & 28 & 40,6 & & \\
\hline \multicolumn{13}{|l|}{$\begin{array}{c}\text { Repetiu alguma } \\
\text { série }\end{array}$} \\
\hline Sim & 4 & 36,4 & 7 & 63,6 & $0,522^{2}$ & & 2 & 18,2 & 9 & 81,8 & $0,321^{2}$ & \\
\hline Não & 35 & 50,0 & 35 & 50,0 & & & 25 & 35,7 & 45 & 64,3 & & \\
\hline \multicolumn{13}{|l|}{$\begin{array}{l}\text { Desempenho } \\
\text { escolar }\end{array}$} \\
\hline Abaixo da média & 7 & 43,8 & 9 & 56,3 & $0,784^{2}$ & & 4 & 25,0 & 12 & 75,0 & $0,559^{2}$ & \\
\hline $\begin{array}{c}\text { Média ou acima da } \\
\text { média }\end{array}$ & 32 & 49,2 & 33 & 50,8 & & & 23 & 35,4 & 42 & 64,6 & & \\
\hline \multicolumn{13}{|l|}{ Fonoaudiólogo } \\
\hline $\operatorname{Sim}$ & 3 & 42,9 & 4 & 57,1 & $1,000^{2}$ & & 3 & 42,9 & 4 & 57,1 & $0,681^{2}$ & \\
\hline Não & 36 & 48,6 & 38 & 51,4 & & & 24 & 32,4 & 50 & 67,6 & & \\
\hline \multicolumn{13}{|l|}{ L. estrangeira } \\
\hline Nenhum & 18 & 46,2 & 21 & 53,8 & $0,825^{2}$ & & 10 & 25,6 & 29 & 74,4 & $0,238^{2}$ & \\
\hline Língua estrangeira & 21 & 50,0 & 21 & 50,0 & & & 17 & 40,5 & 25 & 59,5 & & \\
\hline \multicolumn{13}{|l|}{ Musicalização } \\
\hline Nenhum & 25 & 44,6 & 31 & 55,4 & $0,471^{2}$ & & 16 & 28,6 & 40 & 71,4 & $0,207^{2}$ & \\
\hline Musicalização & 14 & 56,0 & 11 & 44,0 & & & 11 & 44,0 & 14 & 56,0 & & \\
\hline
\end{tabular}

Legenda: TPFS4: Teste Padrão de Frequência com 4 Sons (Símbolo); TPFN4: Teste Padrão de Frequência com 4 Sons (Nomeação); TDE: Teste de Desempenho Escolar; L. estrangeira: Língua estrangeira.

${ }^{1}$ Teste Qui-quadrado de tendência; ${ }^{2}$ Teste exato de Fisher. 


\section{DISCUSSÃO}

De acordo com a literatura ${ }^{19}$, as dificuldades escolares tem sido objeto de estudos, pois afetam um número cada vez maior de estudantes brasileiros, sendo que, o estudo da audição e sua correlação com o desenvolvimento educacional é um campo de imensa importância na busca de explicações e intervenções nas dificuldades escolares.

Ao considerar que o processamento temporal está intimamente relacionado ao desenvolvimento adequado da linguagem ${ }^{4}$, o presente estudo corrobora a literatura ${ }^{7}$, quando indica a necessidade da inclusão de procedimentos que avaliem os aspectos temporais durante a avalição do Processamento Auditivo ${ }^{4,20,21}$, uma vez que conhecer as habilidades de resolução e ordenação temporais pode auxiliar na construção de programas de intervenção ${ }^{15,22}$.

Dentre os procedimentos utilizados para avaliação da resolução temporal, existe o teste GIN (Gaps in noise). De acordo com a literatura ${ }^{23}$, esse foi publicado em 2005 e foi utilizado somente em crianças normais, visando o estabelecimento de critérios de normalidade ${ }^{4}$ e em adultos, para comparação de desempenho das orelhas direita e esquerda ${ }^{1}$.

Nesse estudo, tanto a média dos limiares de gap, quanto a média das porcentagens de acertos do gap evidenciaram que os limiares encontrados foram semelhantes aos encontrados na literatura, em indivíduos normais, tanto crianças ${ }^{23}$, quanto adultos ${ }^{1,3}$.

Ao analisar os dados referentes à associação entre os testes GIN e TDE (Tabela 1), pode-se observar que não houve evidência de significância estatística ao se comparar o desempenho no teste com a classificação dos grupos do TDE. De acordo com a literatura ${ }^{23}$, um indivíduo, em que o sistema auditivo central não está sendo capaz de detectar intervalos breves de silêncio (características de certas consoantes) e perceber as informações corretamente, pode apresentar suas habilidades de leitura e de linguagem prejudicadas. Porém, o presente estudo não corroborou com tal afirmação, pois foi observado que a maioria dos adolescentes classificados no grupo III, com desempenho inferior nos subtestes de leitura (100\%) e escrita $(86,7 \%)$ do TDE obtiveram desempenho normal no GIN.

Em relação às variáveis preferência manual, dificuldade de audição, repetência escolar e aprendizado de uma segunda língua (Tabela 1) não houve significância estatística. Vale ressaltar que na literatura, não foram encontrados estudos que realizassem essas associações. Além disso, observou-se no presente estudo que, as habilidades de resolução temporal da população não foram influenciadas, mesmo que indiretamente, pelo aprendizado musical. Não foi encontrada diferença com significância estatística no desempenho dos indivíduos que realizaram atividade de musicalização se comparados aos que não realizaram. Este dado não corrobora a literatura ${ }^{3}$, uma vez que nesta sugeriu-se a hipótese de que a habilidade de resolução temporal encontra-se mais desenvolvida em músicos, ou seja, trabalhar com a musicalização pode requerer uma percepção auditiva mais refinada. Cabe ressaltar que, em nosso estudo não foram avaliados o tempo ou o grau de experiência musical dos indivíduos. A variável musicalização foi elencada apenas a partir de uma pergunta do questionário de caracterização da amostra.

Já em relação à variável sexo, os achados não corroboram parte dos estudos descritos na literatura $^{5,24}$, uma vez que referenciam diferença com significância estatística entre os gêneros masculino e feminino, no qual o sexo masculino apresenta limiares inferiores, ou seja, pior desempenho. Porém corrobora outras pesquisas que relatam não haver diferença de desempenho com significância estatística, quando comparados os gêneros ${ }^{1,4,23}$.

É importante ressaltar que, de acordo com a literatura ${ }^{1,3,21}$, não há diferença com significância estatística nas respostas do GIN quando comparadas às orelhas direita e esquerda. Assim como, não há diferença com significância estatística entre as faixas-teste do GIN. Tal afirmação justifica a escolha pela aplicação de somente uma faixa-teste durante a realização deste estudo $3,21,24$. No entanto, vale ressaltar que, em um estudo da literatura ${ }^{4}$ foi observado que a primeira faixa-teste apresentou porcentagem maior de identificações de gap com significância estatística em relação a segunda faixa-teste.

Durante a análise dos resultados observou-se que a variável "acompanhamento com fonoaudiólogo" apresentou evidência de significância estatística, em que estudantes que realizaram ou realizam fonoterapia apresentaram desempenho inferior no Teste GIN. Tal achado pode ser justificado pelo fato de que os sujeitos que realizam acompanhamento com fonoaudiólogo possuem alterações ou constituem grupo de risco para alterações da comunicação humana, incluindo o Processamento Auditivo. Visto que, de acordo com a literatura ${ }^{23}$, problemas no desenvolvimento da fala, linguagem, aprendizagem e socialização podem ser acarretadas por alterações no Processamento Auditivo.

A literatura especializada ${ }^{9}$ afirma que a percepção dos aspectos temporais do som desempenha papel crucial na aquisição da leitura e da escrita.

Além da resolução temporal, sabe-se que a ordenação temporal (capacidade de reconhecer, 
identificar e ordenar estímulos acústicos, de acordo com sua ordem de apresentação) também é considerada uma das funções mais importantes do sistema nervoso central, uma vez que a fala e a compreensão da linguagem, são dependentes da capacidade de se trabalhar com a sequência sonora ${ }^{25}$.

A literatura descreve diversos estudos que utilizaram os Testes Tonais de Padrão de Frequência - Pitch Pattern Test (TPF) ${ }^{8,9,25-31}$ e os Testes Tonais de Padrão de Duração do Som Duration Pattern Test (TPD) ${ }^{9,15,25,26,28,31,32-39}$ para avaliação da ordenação temporal. A aplicação de ambos os testes, de acordo com a literatura ${ }^{25}$, é benéfica para avaliação dos sujeitos, pois, embora esses testes possuam construção similar, eles avaliam diferentes processos. Nesse estudo, ao se comparar o desempenho dos adolescentes nos TPF e TPD (Tabelas 2 a 5), pode-se observar que, a média de acertos no Teste Padrão de Duração com 3 ou 4 sons ( 9,3 acertos), foi melhor se comparada a média no Teste Padrão de Frequência (5,1 acertos). Isso porque, de acordo com a literatura pesquisada ${ }^{26}$, existe a hipótese de que a fonética da língua portuguesa possibilite melhor desempenho na resolução de duração se comparada a resolução de frequência, uma vez que os fonemas no português possuem uma distância (em termos de acústica) maiores que outras línguas estrangeiras.

Ainda durante a aplicação do TPD, os escolares relataram que o parâmetro tempo é mais fácil de ser percebido, assim como os conceitos de longo e curto. O desempenho melhor apresentado pelos sujeitos, na presente pesquisa, não corrobora outro estudo da literatura ${ }^{26}$ em que foram observados desempenhos semelhantes entre TPD e TPF para o grupo de brasileiros.

A análise estatística correlacionou os limiares auditivos dos Testes Padrão de Frequência e Padrão de Duração, considerando as variáveis: sexo, idade, preferência manual, dificuldade de audição, repetência escolar e utilização de medicamentos (Tabelas 2 a 5). Não foram observadas associações com significância estatística relacionadas às variáveis supracitadas. No que diz respeito ao sexo e idade, foram encontrados registros na literatura consultada ${ }^{15,25,28}$ corroborando a ausência de significância estatística, visto que o desenvolvimento e a maturação das estruturas responsáveis para realização das tarefas solicitadas nos testes igualam-se ao desempenho de um adulto a partir dos 9 anos de idade, não alterando após os 11 anos.

Foi observado também no presente estudo, que a variável musicalização não exerce influência com significância estatística no desempenho tanto do TPF e do TPD (Tabelas 2 a 5). Tal achado corrobora um estudo da literatura ${ }^{26}$ em que os autores verificaram que somente a discriminação de frequência é influenciada pelo aprendizado da música, tal fato não é verificado na discriminação da duração dos sons. Em relação à variável "aprendizado de uma língua estrangeira" a literatura ${ }^{26}$ aponta para mudanças anatômicas, morfológicas e comportamentais do cérebro durante o processo. Porém, tal aprendizado não influenciou o desempenho em ambos os testes.

Não foram encontradas, na literatura científica, pesquisas que relacionassem 0 desempenho de adolescentes nos Testes de Padrão de Frequência e Duração com o resultado do Teste de Desempenho Escolar. Porém, durante a análise dos dados, observou-se que os integrantes classificados no grupo III no subteste de Aritmética e no escore global do TDE, apresentaram desempenho inferior com significância estatística no Teste Padrão de Duração com 3 sons (símbolos). Tal fato também foi observado nos indivíduos pertencentes ao grupo III no subteste de Escrita. Esses apresentaram desempenho baixo com significância estatística no Teste Padrão de Frequência com 3 sons (nomeação) e com 4 sons (nomeação e símbolos). Ainda em relação aos adolescentes classificados no grupo III no escore global do TDE, observou-se respostas inferiores com significância estatística, se comparados aos integrantes dos outros dois grupos no Teste Padrão de Frequência com 4 sons (nomeação).

Foi observado também que na modalidade "nomeação" tanto no Teste Padrão de Duração, quanto no Teste Padrão de Frequência os alunos apresentaram maior dificuldade. Essa dificuldade pode ser explicada, de acordo com a literatura ${ }^{8}$, pela necessidade de integração inter-hemisférica dos estímulos na resposta verbal, o que não acontece na resposta não-verbal (símbolo). Essa afirmação nos permite sugerir que esses indivíduos podem apresentar dificuldade em outra habilidade do processamento temporal, a integração auditiva. Outra possibilidade encontrada na literatura ${ }^{6}$ para justificar esse achado, está no fato das respostas verbais parecerem mais difíceis em crianças com problemas de linguagem, uma vez que a nomeação envolve processos cognitivos mais elaborados, como atenção, definição do conceito para representação do estímulo percebido e movimentos articulatórios adequados para pronúncia da palavra selecionada.

Além disso, nesses testes os indivíduos classificados como "desempenho médio" nos subtestes de leitura ou escrita do TDE demonstraram resultados inferiores com significância estatística ao serem comparados com os indivíduos classificados como 
"desempenho superior". Esse achado corrobora a literatura especializada ${ }^{6}$ no que diz respeito ao consenso de que alterações nos aspectos temporais são observadas em sujeitos com problemas na aquisição da leitura e escrita.

Ainda de acordo com a literatura ${ }^{35,36}$, alterações no Processamento Auditivo podem apresentar diversos sintomas que interferem na aprendizagem, dentre eles, dificuldade de leitura e escrita e inabilidades para matemática. Este estudo corrobora tal afirmação, uma vez que adolescentes classificados como "desempenho médio" no subteste de Aritmética do TDE apresentaram diferença estatisticamente significante quando o tipo de resposta solicitado no Teste Padrão de Duração com 3 sons foi "símbolo". Em outra literatura ${ }^{37}$, foi observada a associação entre o desempenho em aritmética e as dificuldades atencionais. Dentre diversos estudos citados, foi relatada uma pesquisa ${ }^{38}$ realizada com estudantes do primeiro ano de alfabetização em que foi encontrada diferença com significância estatística entre os alunos com e sem dificuldades atencionais no subteste de Aritmética do TDE.

Por último, foi encontrado um estudo ${ }^{39}$ que destacou a influência do desenvolvimento biológico sobre o processo de ensino-aprendizagem. Nesse artigo foi citada uma pesquisa ${ }^{40} \mathrm{em}$ que, ao estudar a aprendizagem em matemática, observou-se a influência do aspecto comportamental no desempenho dos estudantes, ou seja, quando as atitudes dos estudantes são favoráveis em relação a um conteúdo, o aumento da motivação permite maior concentração durante o processo de aprendizagem.

Sabe-se que atualmente no Brasil existem avaliações comportamentais que diagnosticam as habilidades auditivas temporais. No entanto, apesar dos diversos testes diagnósticos, não há disponível nenhum programa sistemático de avaliação do Processamento Auditivo em escolares. A falta de divulgação nas escolas desse tipo de abordagem, assim como os diagnósticos equivocados em crianças com dificuldades acadêmicas são as consequências diretas ocasionadas pela falta desse tipo de instrumento ${ }^{41}$.

Este estudo ao relacionar o desempenho escolar com os testes que avaliam o processamento auditivo temporal buscou ressaltar a importância de um diagnóstico precoce de alterações do Processamento Auditivo. Esse poderia proporcionar aos profissionais envolvidos com a aprendizagem escolar um meio de minimizar as dificuldades enfrentadas pelos escolares, por meio de uma terapêutica mais efetiva, evitando a repetência escolar. Os resultados apresentados nesse estudo sugerem a necessidade de estimulação das habilidades auditivas de Processamento Temporal no ambiente escolar.

Apesar das contribuições do presente estudo, vale ressaltar as limitações encontradas durante a realização do mesmo. Pode-se citar a utilização da amostra de conveniência em lugar da amostra populacional, o número desigual de estudantes dos dois gêneros e por último e não menos importante, a ausência de uma avaliação audiológica periférica formal para detectar alterações auditivas leves. Vale ressaltar que, apesar de se ter conhecimento sobre a importância da avaliação da audição periférica formal, a mesma não foi realizada, pois como se sabe, nem sempre é possível a realização desse tipo de avaliação no ambiente escolar, sendo a aplicação de testes em campo livre uma alternativa para avaliação de estudantes em seu ambiente de aprendizagem.

\section{CONCLUSÃO}

A análise dos dados revelou que há correlação entre desempenho escolar e aspectos temporais auditivos. Cabe ressaltar que os resultados dos testes que avaliam a ordenação temporal complexa (Teste Padrão de Frequência e Teste Padrão de Duração) apresentaram relação com o desempenho do TDE. Porém o mesmo não ocorre com o teste que avalia resolução temporal (GIN), o qual, não foi influenciado pelo desempenho escolar (TDE). A maior correlação encontrada ocorreu entre o subteste Escrita do TDE e o Teste Padrão de Frequência (TPF). Novos estudos deverão ser realizados para que, dessa forma, possa melhor se compreender a relação entre as dificuldades escolares e o Processamento Auditivo Temporal. 


\begin{abstract}
Purpose: to elucidate the relationship between school performance and temporal aspects of hearing. Method: a descriptive cross-sectional convenience sample composed of 82 students, aged 11-13 years, 43 females, and 38 males in the 6th grade at a private funding school in the Metropolitan Region of Belo Horizonte. Each student responded to one form of sample characterization, performed the Academic Performance Test and was submitted to tests that evaluated the temporal auditory aspects (Duration Pattern Sequence, Pitch Pattern Sequence and Gap-in-Noise). Results: the tests that assess auditory temporal aspects were not affected by the variables gender, age, music activity performance and, hand preference. The speech therapy variable was statistically significant on Gap in Noise Test. The subtests of the Educational Achievements' Test had the greatest influence on the Duration and Frequency Pattern Sequence performance were writing followed by reading and arithmetic. Conclusion: data analysis revealed a correlation between school performance and temporal aspects of hearing. It should be noted that the tests that evaluate the temporal ordering tasks (Frequency Pattern Sequence and Duration Pattern Sequence) are influenced by the Academic Achievement Test performance. However this does not occur with the test that evaluates the temporal resolution (Gap-in-Noise).
\end{abstract}

KEYWORDS: Hearing; Auditory Perception; Hearing Tests; Students

\section{REFERÊNCIAS}

1. Samelli AG, Schochat E. Estudo da vantagem da orelha em teste de detecção de gap. Rev Bras Otorrinolaringol. 2008; 74(2): 235-40.

2. Balen SA, Bretzke LM, Mottecy CM, Liebel G, Boeno MR, Gondim LM. Resolução temporal de crianças: comparação entre audição normal, perda auditiva condutiva e distúrbio do processamento auditivo. Rev Bras Otorrinolaringol. 2009; 75(1): 123-9.

3. Zaidan E, Garcia AP, Tedesco MLF, Baran JA. Desempenho de adultos jovens normais em dois testes de resolução temporal. Pró-Fono Revista de Atualização Científica. 2008; 20(1): 19-24.

4. Perez AP, Pereira LD. O Teste Gap in Noise em crianças de 11 e 12 anos. Pró-Fono Revista de Atualização Científica. 2010; 22 (1): 7-12.

5. Ishii C, Arashiro PM, Pereira LD. Ordenação

e resolução temporal em cantores profissionais e amadores afinados e desafinados. Pró-Fono Revista de Atualização Científica. 2006; 18 (3): 285-92.

6. Muniz LF, Roazzi A, Schochat E, Teixeira CF, de Lucena, JA. Avaliação da habilidade de resolução temporal, com uso do tom puro, em crianças com e sem desvio fonológico. Rev CEFAC. 2007; 9(4): 550-62.

7. Queiroz DS, Branco-Barreiro FCA, MomensohnSantos TM. Desempenho no Teste de Detecção de Intervalo Aleatório - Random Gap Detection Test (RGDT): estudo comparativo entre mulheres jovens e idosas. Rev Soc Bras Fonoaudiol. 2009; 14 (4): 503-7.

8. Santos JLF, Parreira LMMV, Leite RCD. Habilidades de ordenação e resolução temporal em crianças com desvio fonológico. Rev CEFAC. 2010; ahead of print.

9. Frota S, Pereira LD. Processos temporais em crianças com déficit de consciência fonológica. Revista Iberoamericana de Educación. 2006; 1 - 11. 10. Redação Terra. Brasil lidera ranking de repetência escolar na América Latina [base de dados na Internet]. [atualizada em: 2010 Jan 20; acesso em 2010 Nov 01]. Disponível em:

http://noticias.terra.com.br/educacao/noticias/0,,OI 4216214El8266,00Brasil+lidera+ranking+de+repet encia+escolar+na+America+Latina.html

11. Neves IF, Schochat E. Maturação do processamento auditivo em crianças com e sem dificuldades escolares. Pró-Fono Rev. de Atualização Científica. 2010; 17(3): 311-20.

12. Stein, LM. TDE: teste de desempenho escolar: manual para aplicação e interpretação. São Paulo: Casa do Psicólogo. 1994.

13. Lizarro, MBT. Processos temporais auditivos em músicos de Petrópolis. [dissertação especialização] São Paulo (SP): Universidade Federal de São Paulo - Escola Paulista de Medicina; 1999.

14. Dibi, VG. Teste de padrão tonal de duração e freqüência sonora e habilidades grafofônicas. [dissertação mestrado] São Paulo (SP): Universidade Federal de São Paulo - Escola Paulista de Medicina; 2000. 
15. Ramos CS, Pereira LD. Processamento auditivo e audiometria de altas freqüências em escolares de São Paulo. Pró-Fono Revista de Atualização Científica. 2005; 17 (2): 153-64.

16. Rezende, AG. Padrões temporais auditivos em escolares da $4^{\underline{a}}$ série do ensino fundamental. [dissertação mestrado]. São Paulo (SP): Universidade Federal de São Paulo - Escola Paulista de Medicina; 2000.

17. Musiek FE, Shinn JB, Jirsa R, Bamiou DE, Baran JA, Zaidan E. GIN (Gaps in Noise) test performance in subjects with confirmed central auditory nervous system involvement. Ear Hear. 2005; 26(6): 608-18.

18. Samelli, AG. O Teste GIN (gap in noise): limiares de detecção de gap em adultos com audição normal. [Dissertação de Doutorado]. São Paulo (SP): Universidade de São Paulo - Faculdade de Medicina; 2005.

19. Lemos, SMA. Processamento auditivo e estressores familiares em indivíduos com dificuldades escolares. [dissertação doutorado]. São Paulo (SP): Universidade Federal de São Paulo - Escola Paulista de Medicina; 2007.

20. Murphy CF, Schochat E. Influência de paradigmas temporais em testes de processamento temporal auditivo. Pró-Fono Revista de Atualização Científica. 2007; 19(3): 259-66.

21. Balen AS, Boeno MRM, Liebel, G. A influência do nível socioeconômico na resolução temporal em escolares. Rev Soc Bras Fonoaudiol. 2010; 15(1): 7-13.

22. Fortes AB, Pereira LD, Azevedo MF. Resolução temporal: análise em pré-escolares nascidos a termo e pré-termo. Pró-Fono Revista de Atualização Científica. 2007; 19(1): 87-96.

23. Balen SA, Liebel G, Boeno MR, Mottecy CM. Resolução temporal de crianças escolares. Rev CEFAC. 2008; 11(supl. 1): 52-61.

24. Samelli AG. O Teste GIN (Gap in noise): limiares de detecção de gap em adultos com audição normal [dissertação doutorado] São Paulo (SP): Faculdade de Medicina da Universidade de São Paulo; 2005.

25. Schochat $E$ et al. Processamento auditivo: comparação entre potenciais evocados auditivos de média latência e testes de padrões temporais. Rev CEFAC. 2009; 11 (2): 314-22.

26. Onoda RM, Pereira LD, Guilherme A. Reconhecimento de padrão temporal e escuta dicótica em descendentes de japoneses, falantes e não-falantes da língua japonesa. Rev Bras Otorrinolaringol. 2006; 72 (6): 737-46.
27. Fortunato-Tavares $T$, Rocha $C$, Furquim $C A$, Befi-Lopes D, Schochat E, Hestvik A, Schwartz R. Processamento Lingüístico e Processamento Auditivo Temporal em Crianças com Distúrbio Específico de Linguagem. Pró-Fono Revista de Atualização Científica. 2009; 21 (4): 279-84.

28. Campos PD et al. Habilidades de ordenação temporal em usuários de implante coclear multicanal. Rev Bras Otorrinolaringol. 2008; 74 (6): 884-9.

29. Elias KMIF, Santos MFC, Ciasca SM, MouraRibeiro MVL. Processamento auditivo em criança com doença cerebrovascular. Pró-Fono Revista de Atualização Científica. 2007; 19 (4): 393-400.

30. Sanchez LM, Nunes FB, Barros F, Ganança MM, Capovilla HH. Avaliação do processamento auditivo em idosos que relatam ouvir bem. Rev Bras Otorrinolaringol . 2008; 74(6): 896-902.

31. Elias KMIF. Doença cerebrovascular na infância e adolescência: estudo das habilidades de processamento auditivo (central). Rev Soc Bras Fonoaudiol. 2009; 14 (1): 152.

32. Liporaci, FD. Estudo do processamento auditivo temporal (resolução e ordenação) em idosos [dissertação mestrado] Rio de Janeiro (RJ): Universidade Veiga de Almeida; 2009.

33. Abdo A, Murphy C, Schochat E. Habilidades auditivas em crianças com dislexia e transtorno do déficit de atenção e hiperatividade. Pró-Fono Revista de Atualização Científica. 2010; 22 (1): 25-30.

34. Meneguello J, Leonhardt DF, Pereira LD. Processamento auditivo em indivíduos com epilepsia de lobo temporal. Rev Bras Otorrinolaringol. 2006; 72(4): 496-504.

35. Lucion CS, Oliveira PR. Transtorno do Processamento Auditivo: características e implicações na aprendizagem. Revista Roteiro. 2010; 35(1): 73-94.

36. Elgelmann L, Ferreira MDC. Avaliação do processamento auditivo em crianças com dificuldades de aprendizagem. Rev Soc Bras Fonoaudiol. 2009; 14 (1): 69-74.

37. Capovilla, AGS, Dias NM. Desenvolvimento de habilidades atencionais em estudantes da $1^{\text {a }}$ à $4^{a}$ série do ensino fundamental e relação com rendimento escolar. Revista da Associação de Psicopedagogia. 2008; 78(25): 198-211.

38. Martins J, Tonelotto JMF. Avaliação da atenção e desempenho escolar no primeiro ano de escolarização . 56를 Reunião Anual de SBPC; 2004. 39. Dal-Farra RA. Interfaces entre a psicologia e a biologia: tecendo saberes na educação. Revista Vidya. 2009; 28(1): 19-32. 
40. Jesus MAS. As atitudes e o desempenho em aritmética do ponto de vista da aprendizagem significativa [dissertação doutorado] Campinas (SP): Universidade Estadual de Campinas; 2009.
41. Simon LF, Rossi AG. Triagem do processamento auditivo em escolares de 8 a 10 anos. Rev Psicol Esc Educ. 2006; 10(2): 293-304.

http://dx.doi.org/10.1590/S1516-18462012005000040

RECEBIDO EM: 26/04/2011

ACEITO EM: 01/11/2011

Endereço para correspondência:

Sulamita da Silva Marcelino Terto

Rua Itaguara, 196 - apto 302 - Floresta

Belo Horizonte - MG

CEP: 31110-240

E-mail: sulamitamarcelino@yahoo.com.br

Rev. CEFAC. 2013 Mar-Abr; 15(2):271-286 